Basic principles in spectro-imaging. Foreword on Marseilles Observatory - 3D spectroscopic methods

\title{
G. Courtès
}

Observatoire de Marseille, 2 Place Le Verrier, 13248 Marseille Cedex 4, France, and Laboratoire d'Astronomie Spatiale du CNRS, Traverse du Siphon,13012 Marseille, France

\begin{abstract}
Focal reducers provide efficient solutions to the drawbacks introduced by the large focal length of direct foci on large modern telescopes. They house a variety of spectroscopic devices, and lead to various families of spectroimagers. Rational filterings, BPM multisimultaneous bandwidths, extreme spectral resolution of the field scanner interferometers (Tully's first design, Taurus, Cigale) and their image reconstruction, as well as the possibility to obtain the spectra of all image elements of the field owing to the new (Tiger and Silfid) integral field spectrographs sometimes combined with Fabry Perot Interferometers (Pytheas) are now within reach. In classical spectrography the focal reducer leads to the optimized size Baranne's white pupil spectrograph.
\end{abstract}

\section{Focal reducers (FR)}

The direct focus of a telescope does not always provide the best observational parameters, especially on very large telescopes having a large focal length and a redundant oversampling of the image element on the detector pixels. Main consequences are, with the limited size of the modern detectors, the lost of the field dimensions and the lack of large solid angle of the beams illuminating the detector. Discussions for the very large telescopes of the future, like VLT, demonstrate basic advantages of the FR and its various spectroscopic equipments but also point out some severe limitations due to the large diameter of these new telescopes leading to excessive focal length and hence to fast aperture ratio FRs. The purpose of this foreword is to summarize the general philosophy of the Marseilles Observatory group. In addition some original solutions are suggested.

\subsection{History}

The focal reducer (FR) (Courtès 1952, Struve 1955, Meinel 1956) increases the detection power of telescopes for faint extended monochromatic sources. Coupled with interference filters and Fabry-Perot interferometers (FP), the FR detected diffuse $\mathrm{H} \alpha$ emission in the Galaxy and in external galaxy interarm regions, (Courtès et al. 1968) with efficiencies more than thousand times (Courtès 1973) that of the Palomar Sky Survey (POSS.E). Owing to the parallel beams 
between the RF collimator and camera, some spectrographic long slit and multislit modes have also been used as well as interferometeric modes (Courtès 1964). Equivalence of detectability has been established between interferential and spectrographic methods (Courtès 1973). The equivalent energetic continuum "bandwidth" is:

$$
d \lambda=\frac{\Delta \lambda}{F}
$$

with $\Delta \lambda$, bandwidth of the filter and $F$, finesse of the FP. The contrast may reach 2500 times that of the POSS (250 $\AA$ ):

$$
d \lambda=\frac{\Delta \lambda(5 \dot{A})}{F(50)}=0.1 \dot{A}
$$

The FR has been exploited only by the Marseilles group during nearly thirty years with adaptation to the $5 \mathrm{~m}$ Palomar telescope and $6 \mathrm{~m}$ Zelenchuk telescope. With the apparition of CCDs the FR is now generalized on most of the large telescopes (Table I in Courtès 1988).

\subsection{Optical principles}

Improvements of the detector illumination The FR maximizes the solid angle $\omega$ of the beams reaching the detector. For instance, the $f$ ratio goes from direct $\mathrm{f} / 8$ Cassegrain to $\mathrm{f} / 1$ : in this case, the detector illumination increases of $(8 / 1)^{2}$, a factor 64 .

Rational use of the interference filters With insufficient filtering the sky background brightness is so strong that only short integration times are possible. One may take several short frames with average bandwidths $(\Delta \lambda \approx 100 \AA$ interference filters) and add them. But narrow filtering improves the object/sky background contrast. For monochromatic emission objects, we favour interference filters of a few $\AA$ wide (Courtès 1964). When observing galaxies, one would like to avoid changing the interference filter for each redshift. Tunable filters of very small interference order (Atherton and Reay 1981) with $p=7$ to 34 and $\Delta \lambda=30$ to $7 \AA$, would be very convenient (These interference filters must be placed in the narrow and constant incidence beams, (Cassegrain or Nasmyth foci) near the focal plane, otherwise the monochromatic entrance pupil should be severely limited (Courtès 1973) especially when tuning by tilting the filter. Similar designs have been developped (Meaburn 1970) (Tully 1974) (Geyer \& Nelles 1984) for various telescopes and EFOSC for the $3.6 \mathrm{~m}$ ESO.

Space Astronomy Only one application : the Wide Field and Planetary Camera (WF/PC) (HST). This instrument images up to a $2.7^{\prime}$ field at $\mathrm{f} / 12.8$, thanks to four pseudo Schmidt cameras free of vignetting. Field lenses are replaced by 4 concave mirrors. Full transmission from UV $(1160 \mathrm{~A})$ to near IR $(11500 \mathrm{~A})$ is achieved. (Fig. 1).

This kind of catoptric design could be used at the $f / 15$ focus of the VLT (between $3000 \mathrm{~A}$ to 11500 and more) An imaging spectrograph (ISARD) from the UV atmospheric cut off has been designed for the $2 \mathrm{~m}$ Bernard Lyot telescope (Lemaitre et al. 1994) of the Pic du Midi. 


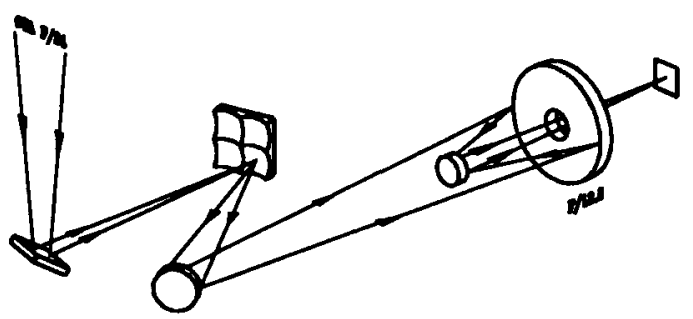

Figure 1. Optical design of the HST Wide Field Camera

Some difficulties arising with the use of CCDs (flatness, reflections) limit in fact around $f / 2$ the $f$ ratios. However, the last FR of the $5 \mathrm{~m}$ Palomar telescope (the PFUEI, Gunn and Westphal 1981) has $\mathrm{f} / 1.4$ for 0.8 " resolution. We shall see that this tendency towards fast $f$ ratios is unavoidable, when one considers the use of FR on telescopes larger than the $4 \mathrm{~m}$ class present generation (reasonable sampling - resolution /nb. of pixels - Shannon rule in case of good $\mathrm{S} / \mathrm{N}$ ).

Table 1 summarizes these conditions for telescopes from 1 to $8 \mathrm{~m}$ diameter, with the hypothesis of $2 \times 15 \mu \mathrm{m}$ pixels for the image diameter of a point like source. One sees that the $f$ ratio $f / 3$ is very well suited to present $3.6 \mathrm{~m}$ telescopes for a good seeing of 0.5 ". For an $8 \mathrm{~m}$ diameter telescope, the $f$ ratio should be $f / 1$ for a seeing of 0.7 ", (the sky brightness should be 9 times higher, per pixel than the one of the present $\mathrm{f} / 3 \quad 3.6 \mathrm{~m}$ telescope CCD camera systems). If one keeps the $f / 3$ as a maximum $f$ ratio the image diameter goes from 30 to $90 \mu \mathrm{m}$, exaggerating the sampling for imagery and reducing the available field by a factor 9 . This drawback is only acceptable for 0.2 " resolution of adaptive optics. On small telescopes, the FR minimizes the focal length, consequently the space resolution. This constraint decreases for large diameter telescopes (Table 1). Extrapolating our experience with the CFHT, the full benefit of a $8 \mathrm{~m}$ telescope should be at $f / 0.7$, for a seeing of 0.5 ", but such a fast $f$ ratio cannot be considered for the reasons noted above (CCD fiatness, reflections and complex optical problems). Fortunately the adaptive optics leads to an easier $f$ ratio of $f / 3.5$ for 0.1 " images, with anyway a severely limited field.

Constraints on the $f /$ ratio of the Very Large Telescope final cameras For the classical use (detection of faint objects) all these extrapolations from $4 \mathrm{~m}$ class telescopes to $8 \mathrm{~m}$ telescopes are full of uncertainties and the best way to select meaningful solutions is to make a direct comparison with the PFUEI instrument on the $5 \mathrm{~m}$ Hale telescope (Gunn and Westphal 1981). With the average good seeing 0.8 " (Bowen 1962) and $15 \mu \mathrm{m}$ pixels, Table 1 gives 0.9 " for 2 pixels of 15 $\mu$ and a FR at $f / 1.4$. Since one hopes a better seeing, 0.5 ", at VLT, one sees (Table 1) that the same $f$ ratio of $f / 1.4$ fits very well with the convincing results of the PFUEI. A FR at VLT equipped with a $\mathrm{f} / 1.4$ camera, would give $9^{\prime} \times 9^{\prime}$ field with $30 \times 30 \mathrm{~mm}$ CCD ( $55 \mu \mathrm{m}$ for 3.7 pixels of $15 \mu \mathrm{m}$ for 0.5 " and 2 pixels for 0.3 " thus keeping full sampling for very good seeing). Only $\mathrm{f} / 1.4$ remains audacious.. Performances extrapoled from PFUEI would be, in imagery around $B_{l i m}=24$ for $\mathrm{FWHM}=20 \mathrm{~A}$ and 2 hours integration time. 
Table 1. Resolution for point like objects images on $2 \times 15 \mu$ pixels

\begin{tabular}{lllll}
\hline Telescope diameter & \multicolumn{4}{c}{ Focal ratios } \\
\hline & f/1 & f/1.4 & f/2 & f/3 \\
1m & $6 "$ & & $3 "$ & $2 "$ \\
2m & $3 "$ & & $1.5 "$ & $1 "$ \\
3.6m ESO-CFHT & $1.6 "$ & & $0.8 "$ & $0.6 "(a)$ \\
5m Palomar & $1.2 "$ & $0.9 "$ & $0.6 "$ & $0.4 "(b)$ \\
6m Zelenchuk & $1 "$ & & $0.5 "$ & $0.3 "$ \\
8m VLT & $0.7 "$ & $0.5 "$ & $0.35 "$ & $0.25 "(c)$
\end{tabular}

(a) Optimal focal reducer (Georgelin 1987)

(b) Compromise Palomar Focal Reducer PFUEI (Gunn \& Westphal (1981)

(c) Possible compromise for VLT commun use between $f / 1.4$ and $f / 2$. The exceptional 0.25 " (adaptative optics) could use f/3.

\subsection{The peculiar case of the VLT, 30' feld, serendipity modes.}

The Ritchey-Chretien $\mathrm{f} / 15$ configuration of the VLT Cassegrain or Nasmyth foci provides a 30' field (nearly 80 times in sky surface the classical FR). It is difficult to imagine that most of this fantastic imagery information should be lost. We give (Fig. 5 in Courtès 1988) an example of four FR working in serendipity mode during the integration time of the central auxiliary instrument (FR or any small field instrument, slit spectrograph, photometer etc... See also Seifert et al. 1994 at this Colloquium). This serendipity mode could be used for routine surveys taking any opportunity during the field center programmed observations.

Segmented pupil Another way to keep these new fantastic field conditions is a segmented pupil system. In the case of the VLT, if one cuts into 6 identical segments, each of them corresponds to a $3.2 \mathrm{~m}$ equivalent pupil (Fig. 6 in Courtès 1988). With this design, simultaneous field imagery in six different colours is possible. This pupil reduction brings back to the favourable case of Table 1: $3.6 \mathrm{~m}$ class telescope and FR at $\mathrm{f} / 2$. Analogy with CFHT CCD performances with oversampling of the same order $(0.11$ "/pixel) leads to a limiting magnitude $B_{\text {lim }}=26$ (Harris 1988) but with the CCD of $30 \times 30 \mathrm{~mm}(2000 \times 2000$ pixel $)$, the field should be however $8 \times 8^{\prime}$. It is known that the pupil segmentation provides a slight improvement of the image quality (Lelièvre \& Arnaud 1988). The coaddition of the six images will certainly reach the quality and the limiting magnitude of a conventional direct frame on the six channels (objective-prism could equip each bandwidth with the corresponding sky background rejection).

\section{Focal reducers and Fabry-Perot - Possible developments}

One is always dependant of the same f ratio constraints (the higher illumination specifications of Table 1).

FR with Fabry-Perots (FP) provide a complete solution for detection imagery as well as for ionized gas radial velocity field studies. 


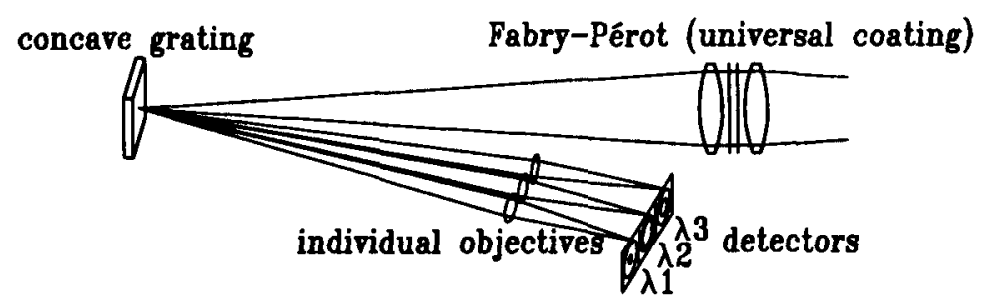

Figure 2. A BPM filter combined with a Fabry-Perot

\subsection{Single bandwidth}

The use of narrow interference filters (see above) in series with FP, gives the best contrast for a faint monochromatic extended source, ( $E$ galaxies: Plana \& Boulesteix, this colloquium). The field scanner interferometers (Tully 1974), Taurus (Taylor \& Atherton 1980), Cigale (Boulesteix et al. 1983) require the same precautions. However it can be useful to sacrifice the spatial resolution (faster focal ratio, then smaller focal length) in order to obtain a better sensitivity and a larger field for extended sources. A good example of this, is the study of the interstellar gas over large fields in the Milky Way (Marcelin et al., Rosado et al. this Colloquium) as well as the observations with telescopes used in poor seeing conditions. Solutions have been experienced on telescopes from $3 \mathrm{~cm}$ (very wide fields, Sivan 1974, Caplan et al. 1985), $36 \mathrm{~cm}$ (le Coarer et al. $1992)$ to $600 \mathrm{~cm}$ diameter (Boulesteix et al. 1983).

\subsection{Multibandwidths}

With FP field scanners, the spectral range is limited to a few lines of close wavelengths. This is not suitable for physical studies of the interstellar gas where emission line intensity ratios are the essential diagnostic tools. It is thus interesting to consider the grating simultaneous multiband filter (BPM filter) (Courtès 1964, Courtès \& Viton 1965, Bonnet \& Courtès 1962), rare solution to provide simultaneous images in different bandpasses in the same condition of atmospheric transmission. The optical BPM design in series in the input common beams with a FP with wide spectral range coating (Pelletier this Colloquium), is shown on figure (2). It would give interference rings of each selected monochromatic image $\lambda_{1} \lambda_{2} \lambda_{3}$ etc.... At the time of the VLT, when CCD mosaics will be common use, this instrument has the merit, with its multiplex qualities to require only one delicate component, the scanning Fabry-Perot. This new design, equipped for instance with a wide free spectral range (f.s.r.) interferometer like the Hawaii Imaging Fabry-Perot, Interferometer (HIFI) (Bland \& Tully 1989), (Veilleux this colloquium) could be the ultimate simultaneous monochromatic wide and narrow lines spectrophotometer. The possibility of a high "finesse" /itF with 7 layers coatings and flatness $\lambda / 200$ of the two plates opens new ways in the application of the Jacquinot demonstration of the considerable efficiency of the etalon compared with the grating spectrographs (Jacquinot 1958). The HIFI interferometer reaches the largest possible f.s.r., the BPM should extend 
to the total spectral range this already remarkable optimization. It should be perfectly adapted to the active nuclei studies and in general to emission lines of the interstellar medium.

\subsection{Tilt}

The tilt of a FP etalon opens the possibility to obtain an uniform distribution of pseudo parallel fringes over the whole field (first recognition of radial velocities or line profile variations or discontinuities). Larger collimator focal length decreases, for a given astronomical field, the curvature of the fringes (Courtès 1964). The line width/pixel ratio (the sampling function) $A(\lambda)$ would become nearly constant, the price to pay is the diameter of the FP.

3. Focal reducers, Integral Field Spectrographs (IFS), BPM, and White Pupil Spectrographs

The challenge is to provide simultaneous imagery at any wavelength extracted from the spectral range together with the individual spectra (or at least the spectral signature) of each image element defined by the size of the telescope and a subarcsecond seeing (Courtès 1982; Courtès et al. 1987). Another step is to reach, owing to adaptive optics, the highest resolution limit (0.2") (Sayede 1991).

\subsection{IFS and Speckle interferometres}

At last, the ultimate step would be the integral field spectrography of the speckles. These highly resolving solutions are obviously dependant on the brightness of the source. Speckle interferometry, requires in addition extremely short integration times (few $10^{-2} \mathrm{sec}$ ). The final focal length must be large enough to sample the speckles. For $2.10^{-2}$ arc sec (the VLT with one telescope) the speckle measures only $12 \mu \mathrm{m}$ in the $\mathrm{f} / 15$ focal plane: there is no need of focal reducer and a minimum of sampling would rather require an enlarger of factor two.

Anyway the f ratio would be about $\mathrm{f} / 30$, perfectly convenient for a BPM application of several simultaneous narrow bandwidths, $\left(\Delta \lambda_{n}\right.$ in direct relation with the $f$ ratio), as Labeyrie did on the Palomar telescope (Gezari et al. 1972). The BPM adapted to the speckle interferometry can be pushed to its extreme possibilities in selectivity with the design proposed by Baranne: using the grating in the very narrow angle beams near the enlarged stellar image, selectivities about 2 angström can be reached (Foy et al. 1992).

\subsection{The white pupil spectrographs: (Baranne 1988)}

A peculiar use of the FR is behind the focal plane of a spectrograph (the spectrum). There the FR superimposes all the dispersed beams in the circular contour of the common exit pupil of the FR. The final camera is then, much smaller and symetrically used at all wavelengths (Fig. p. 1203 in Baranne 1988).

\subsection{Integral Field Spectrograph (IFS)}

Two solutions for the IFS have been considered, 1) the microlens array, 2) the fibers array. We summarize here their characteristics. 


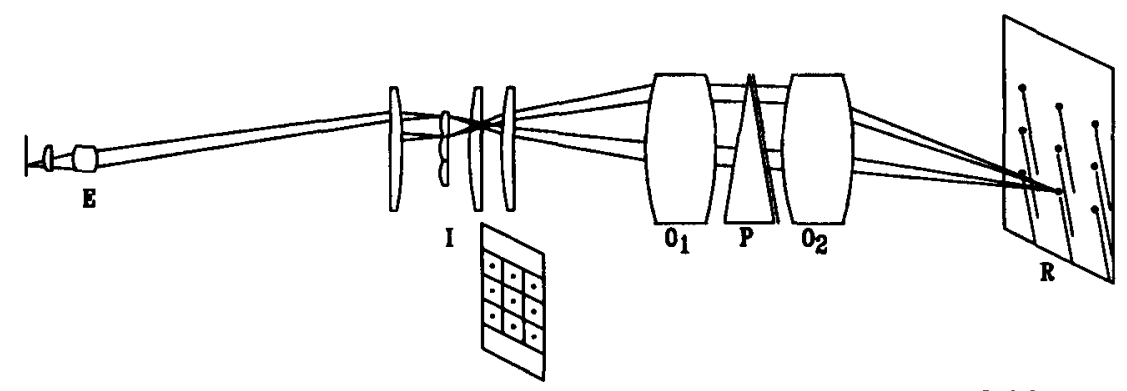

Figure 3. The IFS comprises a telescopic image enlarger, a field lens to provide a telecentric system, the array of lenses and a classical focal reducer (the field lens of it converging the elementary beams issued from the exit pupils to the collimator). A part of the optical path is there in parallel beam and will house the gratings, prisms etc... The final camera forms from the pupils array a matrix of spectra.

Microlens array a) One of the main advantage of the IFS is to isolate in the telescope focal plane the half size of each image element (theorical resolution + seeing) by individual lenses grouped in a $2 \mathrm{D}$ array. The additional dimension will be spectrographic (in a large range of dispersions). The whole image is divided like a mosaic, each lens giving an elementary exit pupil. All the information is contained in the matrix of the image, now transformed in a matrix of pupils - this pupils can be as small as necessary to insure the spectral resolution and scale of the spectrograph - they are in fact playing the role of the slit in any spectrograph. But another advantage of this design is to provide, instead of the somewhat random light repartition in the slits, uniformly illuminated pupils like the well known "Fabry pupils" of the photometrists, thus perfectly defining the instrument function. On the grating one obtains the image of the image element, always half size of the seeing disk, then, the grating is in a rational and uniform illumination.

An adapted software can select any wavelength $\Delta \lambda$ from all spectra and reconstruct the image in this given $\Delta \lambda$, integration of the spectra rebuilds the telescopic image without any deterioration (Bacon, this colloquium, Bacon et al. 1994). The spectra of all image elements are simultaneously obtained and numerous original observations, distant galaxies, nuclei, planetary surfaces are described in this colloquium. A peculiar advantage is, that the geometry of the focal image is preserved in the matrix of the spectra images. The rapid recognition of any spectral features at their right position is easy.

b) The spectral signature in crowded areas (a very new possibility). One of the powerful mean of detection, the grisms, grens etc... used at telescope prime focus are limited by the overlapping of their spectra even for very small dispersion. The IFS method completely avoids this difficulty since its spatial resolution has been adjusted to the seeing conditions. It allows in fact a continuous and simultaneous control of the spectral light distribution of all the image elements of the field. Note that it is an ultimate surface delimitation of the image elements and leads to a peculiarly efficient "moon eliminator". Most of the nebular spectrographs are commonly used with 1 or 2 " slits, the IFS works around 0.3 " 


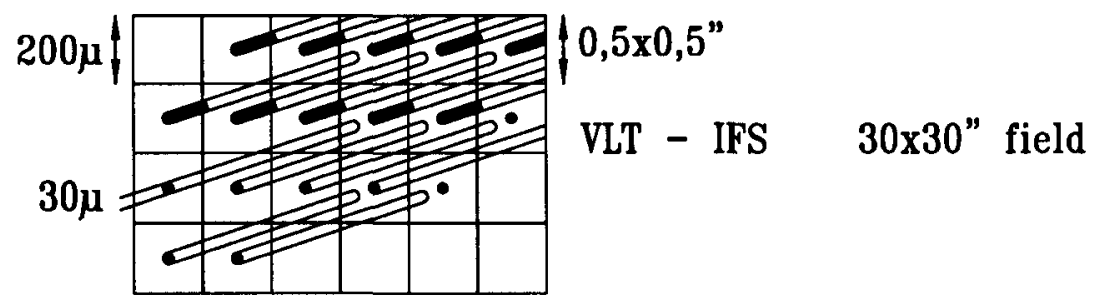

Figure 4. IFS survey instrument for VLT

collecting 9 to 36 times less sky background light per resolved element.

The very well defined "slit function" allows a perfectly controlled small resolution sufficient for spectral signatures $R \leq 10$ always free from overlapping. These small dispersions leads to spectra of very short length $l \leq 20 \mathrm{px}$ about $300 \mu \mathrm{m}$ and hence to an optimized conservation of the telescopic field. Deep surveys of objects at about -2.5 magnitude of the limit of detection can be envisioned with this mode without any loss of morphology or lack of positional recognition (Fig. 4).

c) Integral field spectrograph and Fabry-Perot. A very attractive combination of the IFS and the FP was recently proposed by Georgelin \& le Coarer and described in detail under the name of PYTHEAS (Comte et al. this colloquium). Using a FP it is always necessary to select the f.s.r. by a mono bandpass interference filter, which severely limits the spectral range. Tully's HIFI solution of low order interferometer can extend this spectral range sacrificing the spectral resolution. The PYTHEAS solution uses an IFS like TIGER as an universal premonochromator. Setting a FP in front of the multilenses array, each spectrum of the matrix of spectra on the detector shows a comb of dark fringes along the whole spectral range. The scanning of the FP across "one order" scans all spectral elements. An appropriate software reconstructs the whole spectral range at FP resolution. It is worth noting that the interferometer plates are used on individual very small surfaces thus giving a perfect flatness (zero defect function $\Delta_{D}(\lambda)$ ) and reaching the reflective limit finesse $\mathrm{F}_{R}$ ).

PYTHEAS solves the problem of 3D spectroimagery at high spectral resolution It combines for the first time the FP with a fully adapted premonochromator opening the new field of absorbtion spectra over extended light sources.

Fiber array a) The IFS using fibers (Vanderriest, this colloquium) (Courtès 1982) have also a good uniformity at the output of the fibers with consequently a well defined "slit function". The fibers transmission, at least for the visible range down to the atmospheric UV is convenient for ground based astronomy. However the diameter of the fiber (minimum "slitwidth") is of the order of 100 $\mu \mathrm{m}$ and the output angle corresponds to $\mathrm{f} / 3.2$ and $\mathrm{f} / 2.8$ for some beams in the case of silica (Lemonnier et al. 1993). Both constraints lead to a more complex spectrograph optics. For instance the image on the detector of the output section of the fiber must cover 2 pixels or $30 \mu \mathrm{m}$, the $\mathrm{f}$ ratio of the spectrograph camera will be $f / 1$ since the focal ratio of the collimator must be faster than $f / 3$. 


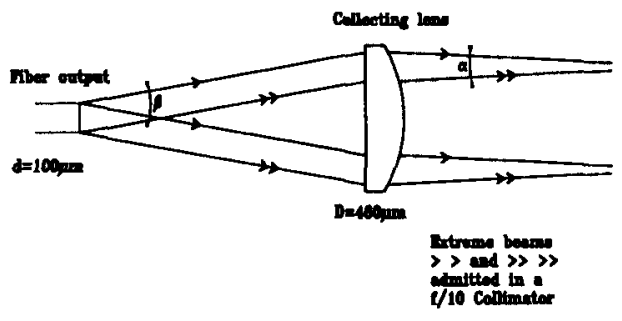

Figure 5. A simple solution for transforming the output fiber $f$ ratio with an increase of the size of the "slit function"

The deviation of flatness of the detectors does not favour such fast $f$ ratio as well as geometric optics difficulties to build the camera. The $\mathrm{f} / 3$ collimator is also difficult to build. Another way is to avoid the fast $\mathrm{f} / 3$ collimator by means of an $f$ ratio transformation near the fiber output, (Fig. 5). A simple converging lens $L$ collects the fiber output beams, then the fratio decreases, $\alpha$ instead of $\beta$, and a more conventional aperture ratio of about $f / 10$ can be achieved. Unfortunately the diameter of the complementary lens $L$ becomes several times the one of the fiber, about $400 \mu \mathrm{m}$, as if the slit width of a spectrograph were several times larger, and there is no other way than to increase the scale of the spectrograph and the ratio of the focal lengths of collimator and camera (about 13) in order to recover a monochromatic image of the "slit" of $2 \times 15 \mu \mathrm{m}=30 \mu \mathrm{m}$. This means again a camera $f$ ratio faster than $f / 1$. One does not see how to get out of this trap. Progress in the fiber manufacturing would perhaps allow to use $50 \mu \mathrm{m}$ diameter fibers and could release near $\mathrm{f} / 2$ the aperture ratio of the final camera.

This unavoidable reduction factor of a limited fiber diameter is the biggest difference between fibers bundles and lens arrays. As a matter of fact the diameter of the micro lens pupils have not this constraint and can be as small as the classical spectrograph slit width.

b) IFS and far UV space research. Another difference is the excellent transparency of the lens array made of MgF2 material in the case of far UV space astronomy as well as that of the micromirrors arrays like the one in our IFS proposal for the Hubble telescope (Courtès et al. 1985).

\section{Conclusions}

A general remark is that IFS (lens or fibers, Tiger and Silfid) provide a 3D simultaneous study (images + spectra) without scanning. The field scanner PF methods (Taurus, Cigale) minimizes the wavelength scanning to one order, with a limited spectral range (various f.s.r) but their "efficiency" can be multiplied by several $\Delta \lambda$ channels owing to the grating filter (BPM) proposed method. It provides the highest resolution. The combination of FP and IFS proposed by Georgelin (PYTHEAS) opens a new original method introducing in the IFS the highest resolutions of the PF with the consequence of a short scanning limited to one FP order but running simultaneously across the whole spectral range. From an optical point of view all these $3 \mathrm{D}$ instruments require fast aperture 
ratios with relatively large field, hence require the highest possible correction of distorsion and chromatic aberration over a large spectral range. These peculiar FR optical difficulties different from those met in conventional spectrograph design have been solved with elegance and simplicity by the major contribution of A. Baranne and G. Lemaitre of Marseilles Observatory.

\section{References}

Amram, P., Marcelin, M., Bonnarel, F., Boulesteix, J., Afanasiev, V.L., \& Dodonov, S.N. 1992, A\&A, 69, 74

Atherton, P.D., \& Reay, N.K. 1981, MNRAS, 197, 507

Bacon, R., Adam, G., Baranne, A., Courtès, G., Dubret, D., Dubois, J.P., Georgelin, Y., Monnet, G., Pecontal, E., \& Urios, J. 1988, ESO conf. VLT instrumentation, Garching, p. 1185

Bacon, R., Adam, G., Baranne, A., Courtès, G., Dubet, D., Dubois, J.PP, Emsellem, E., Ferruit, P., Georgelin, Y.P., Monnet, G., Pécontal, E., Rousset, A. \& Sayède, F. 1994, A\&A, (submitted)

Baranne, A. 1988, ESO conf. VLT instrumentation, Garching, p. 1195 and 1141 Bland, J., \& Tully, B. 1989, AJ, 98, 723

Bonnet, R.M., \& Courtès, G. 1962, Ann. Astrophys., 25, 367

Boulesteix, J., Courtès, G., Laval, A., \& Monnet, G. 1974, Publ. ESO/CERN Conf. on Research Programs for the new large telescopes, Geneva, p. 221

Boulesteix, J., Georgelin, Y.P., Marcelin, M., \& Monnet, G. 1983, SPIE Conf. Inst. Astron. V 445, 37

Bowen, 1962, Private Communication in "Courtès G., 1963, Instruments et Techniques, Bull. Astr. Paris, XXIV, fasc. 2, 239"

Caplan, J., Perrin, J.M., \& Sivan, J.P. 1985, A\&A, 145, 221

le Coarer, E., Amram, P., Boulesteix, J., Georgelin, Y.M., Georgelin, Y.P., Marcelin, M., Joulié, P., \& Urios, J. 1992, A\&A, 257, 389

Courtès, G. 1952, C. R. Acad. Sciences, 234, 506

Courtès, G. 1960, Ann. Astrophys., 23, 115

Courtès, G. 1964, AJ, 69, 325

Courtès, G., \& Viton, M. 1965, Ann. Astrophys., 28, 691

Courtès, G. 1973, Vistas in Astronomy, 14, 81

Courtès, G. 1980, 1er Coll. du Comité Français du Télescope Spatial, Toulouse, Photométrie bidimensionnelle, 241-269

Courtès, G. 1982, IAU Symp. 67, 123

Courtès, G., Cruvellier, P., Detaille, M., \& Saisse, M. 1983, Progress in Optics, Ed. E. Wolf, North Holland Publish. Company, 1-61

Courtès, G., Donas, J., Mauron, N., Vanderriest, C., \& Schneider, J. 1985, LASMATRA, HST Faint Object Camera, Second Generation Instrument, doc. AO.OSSA.4.84, 1-110

Courtès, G., Petit, J.P., Sivan, J.P., Dodonov, S., \& Petit, M. 1987, A\&A, 174, 28 
Courtès, G., Georgelin, Y., Bacon, R., Boulesteix, J., \& Monnet, G. 1987, IAU Symp. Santa-Cruz

Courtès, G. 1988, Proceedings ESO conf. VLT instrumentation, Garching, p. 1141

Foy, R., Foy, F.C., Quatrehomme, F., Thiébaut, E., Blazit, A., Bonneau, D., Tallon-Bosc, I., Tallon, M., Baranne, A., \& Thom, Ch. 1992, Colloque Danemark, Ed. Andersen

Georgelin, Y. 1987, Private Communication

Gezari, D.G., Labeyrie, A., \& Stachmik, R.V. 1972, AJ, 173, L1

Geyer E.H., \& Nelles, B. 1984, IAU Colloque No 79, Garching ESO, 575

Gunn, J.E., \& Westphal, J.A. 1981, SPIE, Solid sate Images for Astronomy, Vol. 290,16

Harris, W.E. 1988, CFHT Information Bulletin 18, 14-15

Jacquinot, P. 1958, Le Journal de physique et le radium, t. 19, p. 223

Le Coarer, E., Amram, P., Boulesteix, J., Georgelin, Y.M., Georgelin, Y.P., Marcelin, M., Joulié, P. \& Urios, J. 1992, A\&A, 257, 389

Lelièvre, G., \& Arnaud, J. 1988, CFHT, Information Bulletin 18

Lelièvre, G., Wlérick, G., Sebag, J. \& Bijaoui, 1994, C. R. Acad. Sciences Paris, 318,905

Lemaitre, G., Comte, G., \& Picat, J.P. 1994 (submitted)

Lemonnier, J.P., Baudrand, J., Casse, M., Cuby, J.G. \& Felenbok, P. 1993, VLT-PLA-FUE-13300-0014, Fuegos design report

Meinel, M. 1956, ApJ, 124, G52

Sayede, F. 1991, Thesis, Lyon University

Seifert, W., Mitsch, W., Nicklos, H. \& Ruppredit, G. 1994, SPIE Symp. on Astronomical telescopes (18 march) Preprint ESO No 63

Sivan, J.-P. 1974, A\&AS16, 163

Struve, 0.1955, Sky and Telescope, XIV, 93

Taylor, K. \& Atherton, P.D. 1980, MNRAS, 191, 675

Tully, B. 1974, ApJ, 27, 415

Vanderriest, C., Courtès, G., Donas, J. 1984, J. of Optics, 15, 237

Westphal, J.A. 1979, WF/PC GSFC - Preliminary design review package (CM 04) (NASA Doc.) 